\title{
Wideband Phased Array Calibration Method for Digital Beamforming
}

\author{
M. Longbrake, L. L. Liou, D. M. Lin, , P. Buxa, J. McCann, T. Pemberton, T. Dalrymple \\ and S. Hary \\ AFRL/RYDR, Building 620, 2241 Avionics Circle, WPAFB, OH 45433-7322
}

\begin{abstract}
Previous study has used chirp signals to characterize the RF response of a multi-channel RF receiver [1]. The results showed a significant channel imbalance. After applying a time-reversal filter, the channel imbalance was remedied and both phase and amplitude were realigned among the channels. In the present study, the RF response of a complete multichannel receiver with antenna was characterized. A 1-8 $\mathrm{GHz}$ phased array antenna was mounted in an anechoic chamber and a chirp signal was used to measure the frequency response of each channel as a function of azimuth angle. Each channel's response was equalized using a time-reversal filter derived from the measured data and a combined beam pattern was formed. Time-reversal filters that steer the beam were also generated and it was found that sidelobe level worsened with larger steer angles. A simple mutual coupling model was proposed to explain the beam pattern feature.
\end{abstract}

\section{INTRODUCTION}

Wideband digital beamforming (DBF) technology has been under intensive development because of its importance in commercial wideband communication and electronic intelligence applications [2,3]. DBF is particularly useful because of its ability to shape the antenna beam pattern in both the spatial and spectrum domain. It enables effective performance in a crowded signal environment by allowing high gain in the direction of the signal of interest while suppressing the gain toward an interferer. Alternatively, DBF allows one to admit the spectrum of the signal of interest while denying the spectrum of the interferer. To achieve DBF's adaptive capacity, the spatial and spectral filter coefficients are computed by solving for a weighing vector applied to the digitized output of the array elements. The weighing vector can be found by solving a convex optimization problem that minimizes the difference between the calculated and the desired spatial and spectral patterns. The basis of the algorithm is an accurate model of the RF response of the array system $[4,5]$ from which to compute the spatial and spectral patterns. The RF response of the phased array can be modeled analytically; however, the task requires accurate component and antenna modeling [6-8] which involves laborious spectrum domain calibrations for each component in the RF chain. This is illustrated in Fig. 1 where there are two time delay components in each channel, one from the free space path and another from the receiver group delay. The free space component is simple to model, but the RF system is very difficult to model.

Previous study [1] discussed a method used for measuring the RF system response. The system in that study is a multi-channel, receive-only system without an antenna. The method used is the timereversal method [9-13]. This paper continues the study with a complete phased array system, that is, antenna elements are included. The RF response as a function of angle of arrival (AOA) was measured. The beam pattern as a function of steering angle will be calculated and discussed.

Section II describes the system, experimental setup and briefly the time reversal method used to measure RF impulse response. Section III shows and discusses the beam pattern results. Finally, the conclusion is given in Section IV.

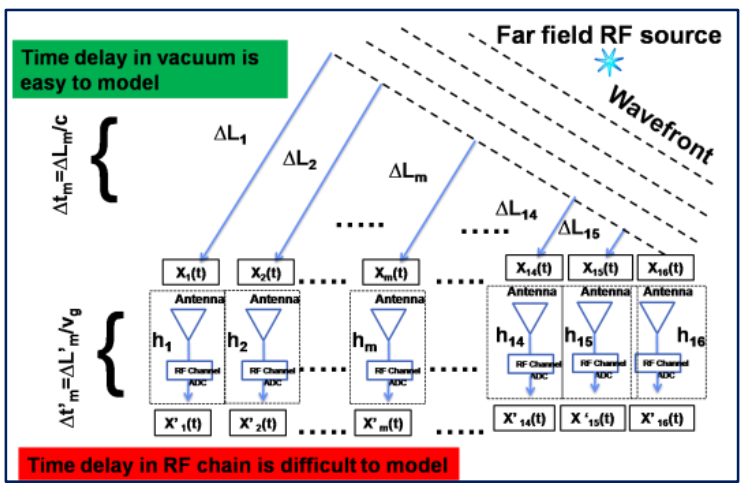

Fig. 1, A schematics of DBF time delay modeling. 


\section{EXPERIMENT}

\section{II.1 System}

The system is a one-dimensional, wideband, 16element phased array covering 1-8 GHz. The spacing between elements is 0.45 inches. It is a channelized system with an instantaneous bandwidth of $500 \mathrm{MHz}$. A tuner system downconverts the input RF frequency to an intermediate frequency band of 750 to $1250 \mathrm{MHz}$. The ADC's sampling rate is $1.333 \mathrm{GHz}$. The system was measured from -60 to 60 degrees in azimuth. A detailed description of the system has also been reported in $[1,14]$.

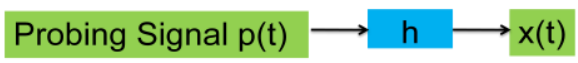

$$
\begin{aligned}
& x(t)=p(t) \otimes h(t) \\
& \text { If } p^{*}(-t) \otimes p(t)=\delta(t) \\
& \text { then } h(t)=p^{*}(-t) \otimes x(t)
\end{aligned}
$$

Probing signal requirement: "Unitary" condition Chirp signal meets the condition

Fig. 2, Method to obtain impulse response using probing signal

\section{II.2 Probing Signal}

To characterize the wideband $\mathrm{RF}$ response in the frequency domain, a set of $\mathrm{CW}$ pilot tones regularly spaced across the bandwidth can be used to measure the amplitude and phase of the frequency response. It is also possible to do this in the time domain. A chirp signal covering the same swath of spectrum is used and the impulse response can be obtained as illustrated in Fig. 2. The chirp signal is given by:

$$
p(t)=\exp \left[j 2 \pi\left(f_{0} t+\frac{B}{2 T} t^{2}\right)\right]
$$

where $\mathrm{f}_{0}$ is the starting frequency, $\mathrm{B}$ is the bandwidth of the chirp, and $\mathrm{T}$ is the duration of the chirp. This signal satisfies the "unitary property" condition, i.e.,

$$
p^{*}(-t) \otimes p(t) \cong \delta(t)
$$

where $\otimes$ is the convolution operator, $*$ indicates the complex conjugate, and $\mathrm{p}^{*}(-\mathrm{t})$ is the time-reversal of the probing signal. It is noted in the previous study that the chirp's baseband bandwidth should be between the Nyquist bandwidth $(1.333 \mathrm{GHz} / 2=$ $666 \mathrm{MHz}$ ), and the instantaneous bandwidth of the system (i.e., $500 \mathrm{MHz}$ ). In this study, we use $666 \mathrm{MHz}$ as the chirp's bandwidth. It is also noted that the chirp method does not require high power, but it requires a large time-bandwidth product. The latter quantity is equivalent to the processing gain in signal processing for increasing S/N. Fig. 3 shows a heuristic example of the importance of this property. When BT is small, the spectrum still resembles a CW pulse. Until BT is large enough, the signal does not excite enough spectrum to be useful. The pulse width of the chirp in our experiment is 20 microseconds and the information content $\left(\mathrm{B}^{*} \mathrm{~T}\right)$ is 13320, which, after processing, yields sufficient information of the digitized impulse response.

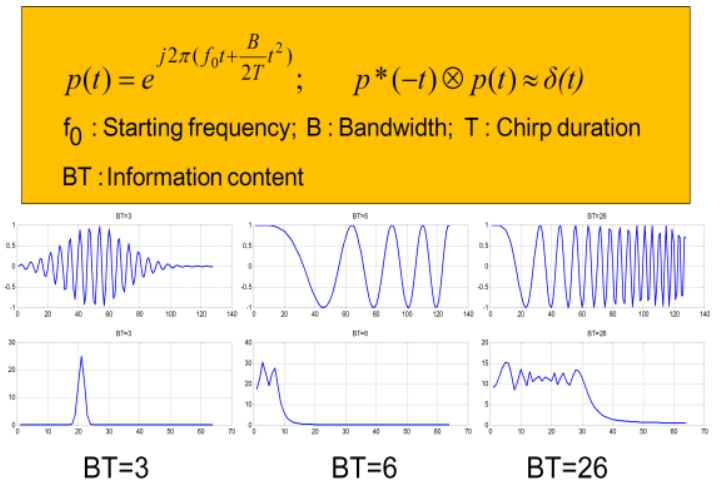

Fig. 3, A heuristic example of the BT product.

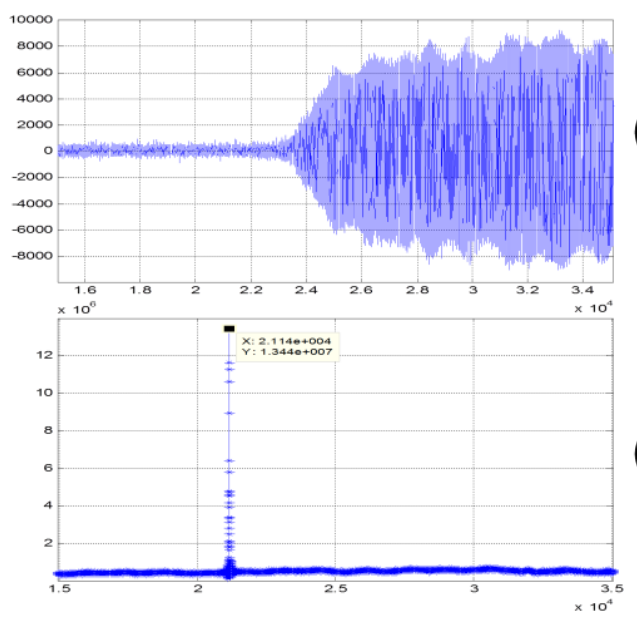

(A)

Fig. 4, (A) A portion of a chirp pulse; (B) Starting point of the chirp 


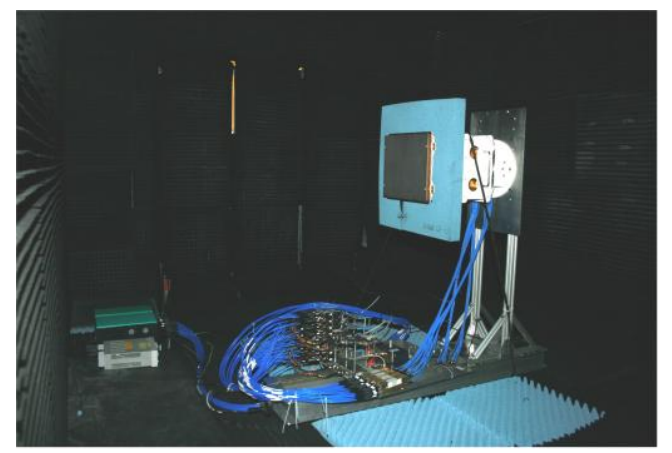

(a)

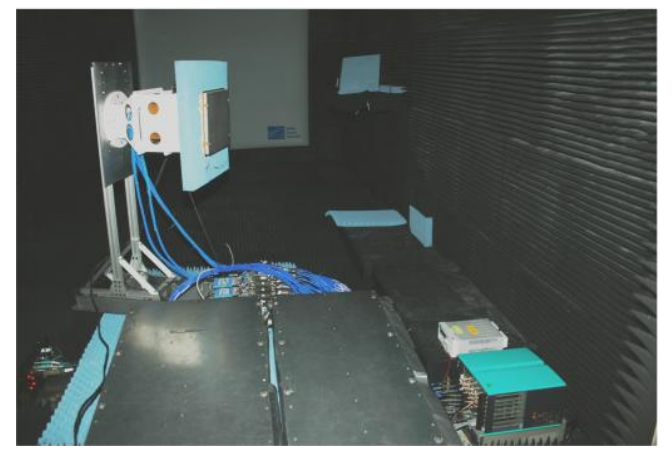

(b)

Fig. 5, (a) Experimental setup showing the phased array antenna panel mounted on a positioned. (b) Transmitting horn antenna and reflector are shown.

A typical measurement in our experiment contains three chirp pulses. The pulse width is 20 microseconds and the pulse repetition interval (PRI) is 30 microseconds. The recorded response due to a pulse input is shown in Fig. 4a. Fig. 4b shows the start of the pulse. The start appears earlier than one would expect from Fig. 4a because the system bandwidth is smaller than the chirp bandwidth.

\section{II.3 Experiment Setup}

The experiment was conducted in a medium size anechoic chamber as shown in Fig. 5. The phasedarray antenna was mounted to an azimuth positioner to provide angle-dependant measurements. The radiation source transmits $\mathrm{RF}$ signals through the feed horn located on the chamber wall. After bouncing off the reflector, the RF plane wave propagates to the phased-array. An arbitrary waveform generator (AWG) sends the probing signal to the feed horn and multi-channel, time-domain data captures are performed for each azimuth angle. Note that the RF path is from the AWG output to the ADC output of the phased array system. Therefore, the raw data of the RF response includes not only the phased array system, but also the medium between the horn and the antenna, including the reflector.

Table I shows the experiment test matrix of the three frequency ranges and AOAs in this study. The RF response was measured at three frequency bands: $3.0-$ 3.5 GHz, 5.0-5.5 GHz and 7.0-7.5 GHz. The AOA dependency is measured from $-60^{\circ}$ to $60^{\circ}$ with an interval of $1^{\circ}$.

Table I, Chirp frequency and AOA plan

\begin{tabular}{|c|c|c|c|}
\hline $\begin{array}{c}\text { Center Freq } \\
(\mathrm{GHz})\end{array}$ & $\begin{array}{c}\text { Chirp BW } \\
(\mathrm{MHz})\end{array}$ & $\begin{array}{r}\text { StartingFreq } \\
(\mathrm{GHz})\end{array}$ & $\begin{array}{c}\text { End Freq } \\
(\mathrm{GHz})\end{array}$ \\
\hline 3.25 & 666 & 2.917 & 3.583 \\
\hline 5.25 & 666 & 4.917 & 5.583 \\
\hline 7.25 & 666 & 6.917 & 7.583 \\
\hline
\end{tabular}

Process $3.25 \mathrm{GHz}$ and $\mathrm{AOA}$ data before conducting others

$$
\begin{aligned}
& \text { Power Level }=(80 \% \text { of FS }) \\
& \text { AOA: }\left[-60^{\circ}: \Delta \phi: 60^{\circ}\right] \text { in degree } \\
& \Delta \phi=1^{\circ} \\
& \text { Total \# of data set }=121 \times 3 \times 1=363
\end{aligned}
$$

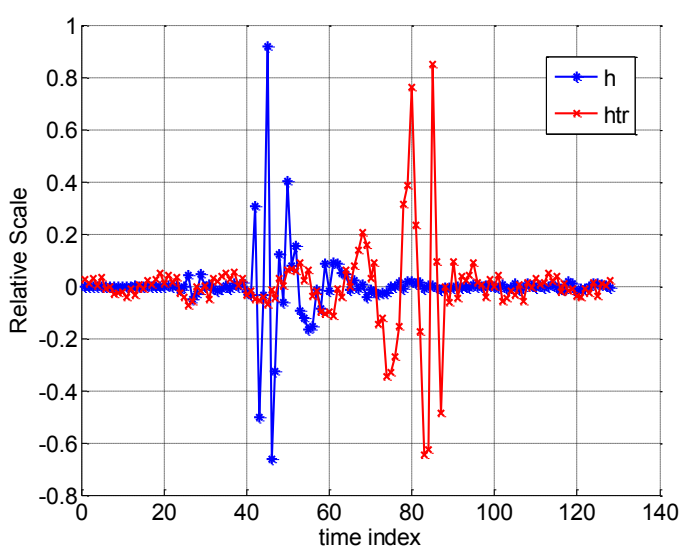

Fig. 6, A typical impulse response (h) and time reversal impulse response $\left(h_{\text {tr }}\right)$.

\section{RESULTS AND DISCUSSION}

\section{III.1 Time Reversal Impulse Response}

The time reversal technique allows the input at the system's entrance point to be generated from the output signal as given by:

$$
x_{\text {ent }}(t)=x_{\text {out }}(t) \otimes h^{\prime}(t)
$$


where $\mathrm{x}_{\mathrm{ent}}$ and $\mathrm{x}_{\mathrm{out}}$ are the signal at the entrance and exit points, respectively, and $h^{\prime}(t)$ is the time reversal impulse response, given by:

$$
h^{\prime}(t)=F^{-1}\left\{H^{*} /|H|^{2}\right\}
$$

where $\mathrm{H}$ is the Fourier transform of the measured impulse response, $h$. A typical $h$ and $h$ ' is shown in Fig. 6. A time reversal filter was computed for each measured angle. Since h' equalizes the amplitude and phase of the channels, using an h' from an angle other than zero effectively steers the beam to that angle.

\section{III.2 Beam Pattern:}

The channel output resulting from the application of a given h' filter can be computed by convolving the measured signal with the h' filter:

$$
x_{\text {ent }}^{f_{0}, \phi_{0}, \phi}(t)=x_{\text {out }}^{f_{0}, \phi}(t) \otimes h_{\phi_{0}}^{\prime}(t)
$$

where $x_{\text {out }}^{f_{0}, \phi}(t)$ is the measured signal with stimulus at frequency $\mathrm{f}_{0}$ and positioner angle $\phi$, and $h_{\phi_{0}}^{\prime}(t)$ is the time reversal filter for steering angle $\phi_{0}$. Note that in this case the stimulus is simply a sinusoid. To plot the beam pattern, the signal power at each positioner angle is found by performing an FFT on $x_{\text {ent }}^{f_{0}, \phi_{0}, \phi}(t)$ and computing the magnitude of the frequency component at $\mathrm{f}_{0}$ :

$$
P_{f_{0}, \phi_{0}}(\phi)=\left|X_{e n t}^{f_{0}, \phi_{0}, \phi}\left(f_{0}\right)\right|^{2}
$$

Figs. 7, 8 and 9 show the beam pattern at a frequency of $7.4 \mathrm{GHz}$ and steering angle of $0^{\circ}, 30^{\circ}$ and $50^{\circ}$, respectively. The ideal beam pattern and the fitted pattern are also shown. The ideal beam patterns are generated directly from the array factor equation, while the fitted results are an attempt to replicate the measured results by introducing mutual coupling to ideal data. This is discussed in the next section. For a steering angle of $0^{\circ}$, the derived beam pattern is close to the ideal beam pattern. The first sidelobe is near $13 \mathrm{~dB}$ down from the main lobe. As the steering angle increases, the derived beam pattern deviates more from the ideal one. When the steering angle is $50^{\circ}$, the largest sidelobe occurs at the $2^{\text {nd }}$ sidelobe, and it is only $4 \mathrm{~dB}$ down from the main lobe. The increase of the sidelobe deteriorates the DBF performance due to dynamic range reduction.

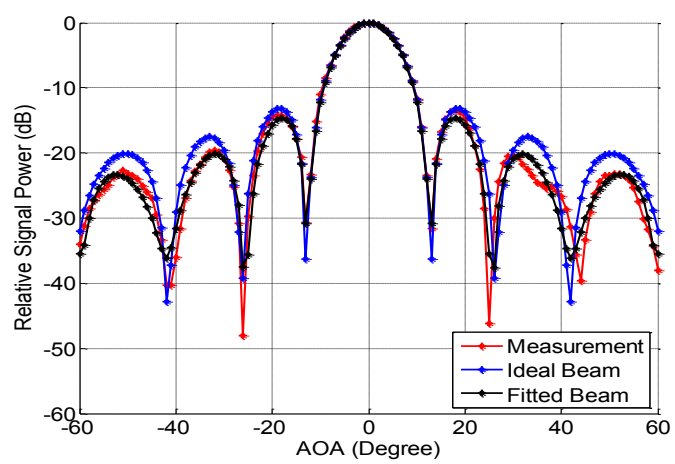

Fig. 7, Beam pattern of frequency $=7.4 \mathrm{GHz}$ and AOA of $0^{\circ}$.

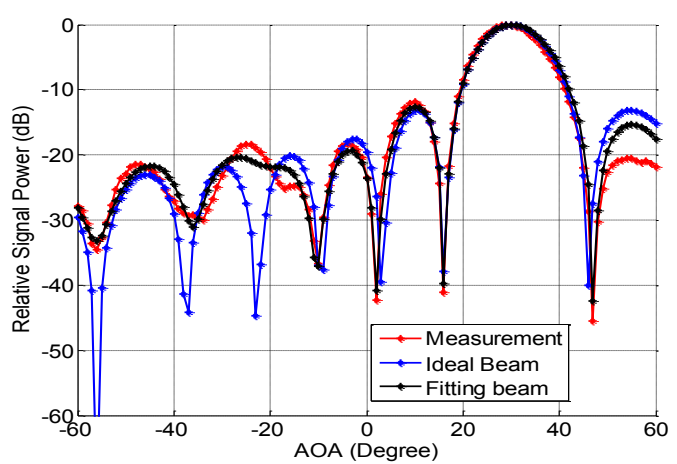

Fig. 8, Beam pattern of frequency $=7.4 \mathrm{GHz}$ and $\mathrm{AOA}$ of $30^{\circ}$

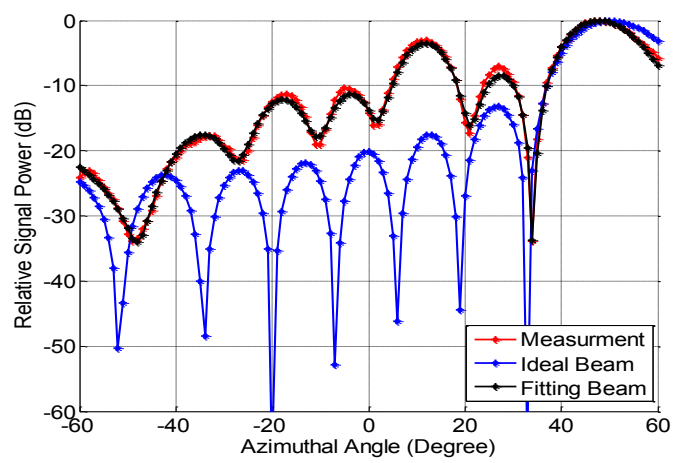

Fig. 9, Beam pattern of frequency $=7.4 \mathrm{GHz}$ and $\mathrm{AOA}$ of $50^{\circ}$.

In addition to sinusoidal stimuli, the beam pattern was also formed with chirp stimuli: 7-7.4 GHz, 5-5.4 $\mathrm{GHz}$ and 3-3.4 GHz. The results are shown in Figs. 
10,11 and 12, respectively, for a steering angle of $50^{\circ}$. The results show that as frequency decreases, the sidelobe degradation improves.

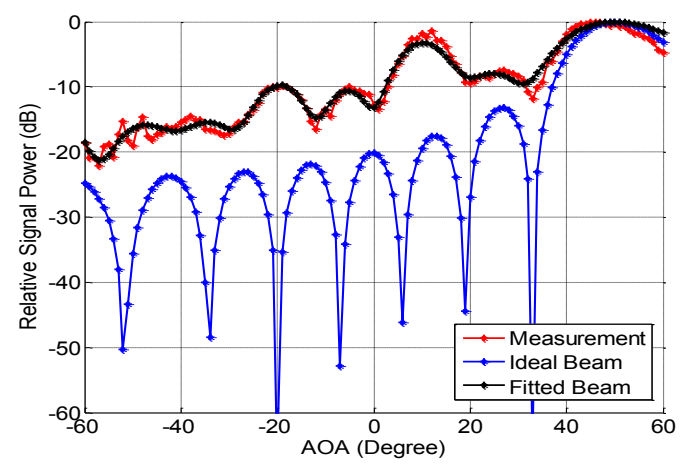

Fig. 10, Beam pattern of a chirp stimuli of 7-7.4 GHz and $\mathrm{AOA}$ of $50^{\circ}$.

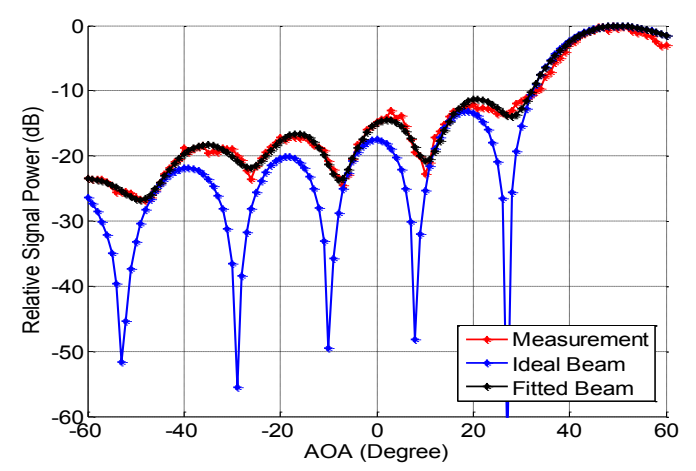

Fig. 11, Beam pattern of a chirp stimuli of 5-5.4 GHz and $\mathrm{AOA}$ of $50^{\circ}$.

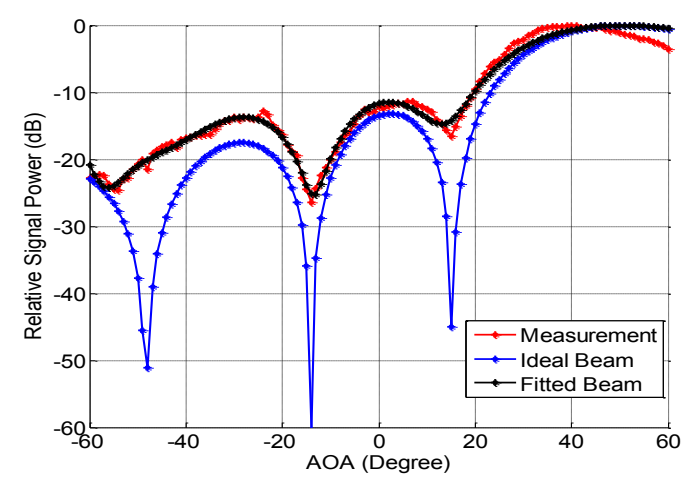

Fig. 12, Beam pattern of a chirp stimuli of 3-3.4 GHz and $\mathrm{AOA}$ of $50^{\circ}$.

\section{III.3 Antenna Coupling Effect}

There are multiple contributions to the received signal that have been ignored in the previous discussion. One is the effect that the chamber (horn, reflector) have on the transmit signal. We assume this effect to be minor since the wideband horn antenna and the reflector is calibrated and supposedly to provide a plane wave when signal reaches the phased array antenna. The primary distortion would be in the frequency domain rather than the spatial domain and corrected by the time-reversal filter. The beam pattern behavior shown in Figs. 7, 8 and 9 is speculated to be due to antenna coupling effects. The design of this wideband phased-array antenna is in contrast to conventional design that tries to reduce mutual coupling between antenna elements. It is designed based on an approach that relies on strong mutual coupling through capacitance between closely spaced dipole antenna elements to reduce the grating lobes and achieve wide bandwidth operation [15]. The mutual coupling affects the beam pattern. It has been shown that the effect causes sidelobe levels to increase as steering angle increases [16,17].

We developed a simple model based on mutual coupling and attempted to find a coupling matrix that generated a pattern similar to the measured data. Assuming a phased array produces a voltage vector when it receives a plane wave with an AOA of $\phi_{0}$, the voltage vector is given by:

$\mathbf{v}_{\phi_{0}}=\mathbf{C s}_{\phi_{0}}$

where $\mathbf{C}$ is the mutual coupling matrix and $\mathbf{s}_{\phi_{0}}$ is the direction vector at $\phi_{0}$, given by:

$$
\begin{aligned}
& \mathbf{s}_{\phi_{0}}=\left[\begin{array}{lllll}
s_{\phi_{0}}^{1} & s_{\phi_{0}}^{2} & s_{\phi_{0}}^{3} & \ldots & s_{\phi_{0}}^{M}
\end{array}\right]^{H} \\
& s_{\phi_{0}}^{m}=\exp \left[j k d_{m} \sin \left(\phi_{0}\right)\right]
\end{aligned}
$$

where $\mathrm{k}$ is the wave number and $\mathrm{d}_{\mathrm{m}}$ is the coordinate of the $\mathrm{m}$-th antenna element. The beam pattern is given by:

$$
P(\phi)=\mathbf{v}_{\phi_{0}}^{H} \mathbf{s}_{\phi}=\mathbf{s}_{\phi_{0}}^{H} \mathbf{C}^{H} \mathbf{s}_{\phi}
$$


The coupling matrix is approximated by a banded matrix taking contributions only from the nearest neighbor. It is given by:

$$
C=\left[\begin{array}{cccccc}
\gamma_{11} & \gamma_{12} & 0 & 0 & 0 & 0 \\
\gamma_{21} & \gamma_{22} & \gamma_{23} & 0 & 0 & 0 \\
\bullet & \bullet & \bullet & \bullet & \bullet & \bullet \\
0 & 0 & \bullet & \bullet & \bullet & 0 \\
0 & 0 & 0 & \gamma_{M^{\prime} 1} & \gamma_{M^{\prime} 2} & \gamma_{M^{\prime} 3} \\
0 & 0 & 0 & 0 & \gamma_{M 1} & \gamma_{M 2}
\end{array}\right]
$$

$M^{\prime}=M-1$

There are 46 complex coefficients in the coupling matrix. There are 121 measured beam patterns, i.e., steering angle is varied from $-60^{\circ}$ to $60^{\circ}$ with an interval of $1^{\mathrm{O}}$. Multidimensional unconstrained nonlinear minimization codes in Matlab were used to best fit the 121 beam patterns with a 92-parameter C matrix. The best-fitted results are also shown in Figs. 7,8 and 9 for a stimulus frequency of $7.4 \mathrm{GHz}$ and steering angle of $0^{\circ}, 30^{\circ}$ and $50^{\circ}$, respectively. Fig. 10,11 and 12 also include fitted data for a steering angle of $50^{\circ}$, and chirp frequency of 7-7.4, 5-5.4 and 3-3.4 GHz, respectively. These preliminary results indicate a reasonable fitting quality, but some of them lack detail agreement. Perhaps this is due to the implied mutual coupling model being too simple to describe the current phased array system. Future study will include a system based on a different antenna design in which mutual coupling is minimized.

\section{SUMMARY}

A phased array system was characterized and impulse response in the RF spectrum was measured. By applying the time-reversal technique, the intra- and inter-channel frequency responses were balanced and corrected. Corrected channel measurements were used to derive the beam pattern. It was shown that the beam pattern deviates significantly from the ideal array factor when the steering angle is off-boresight. The sidelobe level was found to be steering angle dependent, with the sidelobe level increasing as steering angle increases. A simplified model is proposed to depict mutual coupling effects. It is shown that this model can be used to fit the measured steering angle dependent beam pattern. Further study will apply the time reversal calibration technique to an array with less mutual coupling to see if performance with steered patterns improves.

\section{REFERENCES}

[1] L. L. Liou, D. M. Lin, M. Longbrake, P. Buxa, J. McCann, T. Dalrymple, J. B. Tsui and R. Qiu, "Digital Wideband Phased Array Calibration and Beamforming Using Time Reversal Technique", Symposium of Phased Array System and Technology, Boston, MA, October, 2010.

[2] D. D. Curtis, D. N. Spendley and D. Q. Luu, "Current Capabilities of Digital Beamforming", Air Force Research Laboratory Report, Sep. 2007, AFRL-SN-HS-TP-20070011.

[3] J. Tsui, "Special Design Topics in Digital Wideband Receivers", 2010 Artech House, Norwood, MA.

[4] Zhen Hu, Nan Guo, Robert Qiu, Jason Bonior, Lihyeh Liou, David Lin, Matthew Longbrake, Peter Buxa, Thomas Dalrymple, Stephen Hary, James Tsui, "Robust Wideband Beamforming", 2010 NAECON, July 14-16, 2010.

[5] Terry N. Guo, Zhen Hu, Jason Bonior, Robert Qiu, Lihyeh Liou, David Lin, Matthew Longbrake, Peter Buxa, Thomas Dalrymple, Seng Hong, Stephen Hary, and James Tsui, "Wideband Beamforming with Heavily Imbalanced Channels", 2011 NAECON, July 20-22, 2011

[6] J. Wood and D. E. Root (ed.), Fundamentals of Nonlinear Behavioral Modeling for RF and Microwave Circuits, Artech House, 2005.

[7] D. Schreurs, M. O'Droma, A. A. Goacher, and M. Gadringer, RF Power Amplifier Behavioral Modeling, Cambridge University Press, 2009.

[8] A. Raffo, V. Vadala, D. M. M.-P. Schreurs, G. Crupi, G. Avolio, A. Caddemi, G. Vannini, "Nonlinear Dispersive Modeling of Electron Devices Oriented to GaN Power Amplifier Design," IEEE Transactions on Microwave Theory and Techniques, vol.58, no.4, pp.710-718, April 2010.

[9] M. Fink, "Time Reversal of Ultrasonic Fields- Part I: Basic Principles," IEEE Trans. Ultrasonics, Ferroelectrics and Frequency Control, Vol. 39, No. 5, September, 555566, 1992.

[10] F. Wu, J-L Thomas, and M. Fink, "Time Reversal of Ultrasonic Fields- Part II: Experimental Results," IEEE Trans. Ultrasonics, Ferroelectrics and Frequency Control, Vol. 39, No. 5, September, 567-578, 1992.

[11] A. Derode, P. Roux, and M. Fink, "Robust Acoustic Time Reversal with High-Order Multiple Scattering," Physical Review Letters, Vol. 75, No. 23, 4206-4209, 1995.

[12] R. C. Qiu, C. Zhou, N. Guo and J. Q. Zhang, "Time Reversal with MISO for Ultra-Wideband Communications: Experimental Results," IEEE Radio and Wireless Symposium, 499-502, 2006.

[13] H. T. Nguyen, J. B. Anderson, and F. F. Pedersen, "The Potential Use of Time Reversal Techniques in Multiple Element Antenna Systems," IEEE Communication Lett., Vol. 9, No. 1, 40-42, 2005. 
[14] J. Buck, P. Buxa, T. Dalrymple, D. Kuhl, M. Longbrake, J. McCann, D. Spendley, "Transformational Element Level Arrays (TELA) Testbed," AMTA 2008, 16 Nov 2008.

[15] R. C. Taylor, B. A. Munk, T. E. Durham, "Wideband Phased Array Antenna and Associated Methods", US

Patent No: US 6,512,487 B1, Date of Patent: Jan. 28, 2003
[16] H. Steyskal, and J. Herd, "Mutual Coupling Compensation in Small Array Antenna", IEEE Trans. Antenna and Propagation, vol. 38, 1990, pp. 1971-1975. [17] R. Hossa and M. Bialkowski, "Mutual Coupling Compensation in Narrowband Small Linear-Antenna Arrays", Microwave and Optical Technology Letters, vol. 40, pp. 391-396. 